\title{
Adsorção de cobre em amostras de Plintossolo do Estado do Piauí, com diferentes características
}

\author{
Lúcia H. G. Chaves' ${ }^{1}$, Marcos E. B. Brito ${ }^{2}$, Aurean de P. Carvalho ${ }^{2}$, Rossini Daniel ${ }^{2}$, Susane Ribeiro ${ }^{2}$ \& Robi T. dos Santos ${ }^{2}$
}

\begin{abstract}
RESUMO
A capacidade de adsorção de cobre pelo solo é um dado importante em estudos de movimentação de solutos uma vez que, se a capacidade adsortiva de um solo for ultrapassada, o metal ficará potencialmente disponível para ser lixiviado. Neste trabalho se analisam o comportamento da adsorção de cobre face às diferentes características de amostras de Plintossolo e a influência da variação de pH na adsorção do cobre, verificando-se a adequação das equações de Langmuir e Freundlich para descrever a adsorção deste elemento. Soluções de cloreto de cobre foram utilizadas nas concentrações 6, 10, 20, 40, 60 e $80 \mathrm{mg} \mathrm{L}^{-1}$ ajustadas a pH 4,0, 5,0 e 6,0. Os teores de matéria orgânica e a capacidade de troca catiônica das amostras de solo compõem as propriedades que mais influenciaram na adsorção do cobre. Os modelos de Langmuir e Freundlich mostraram-se igualmente capazes para descrever o comportamento do cobre nas diferentes amostras de solo estudadas. A adsorção do cobre variou em função do pH ocorrendo maior adsorção no pH 6,0.
\end{abstract}

Palavras-chave: micronutriente, isotermas, propriedades de solo

\section{Copper adsorption in Plinthosol samples from Piauí State, Brazil, with different characteristics}

\begin{abstract}
Copper adsorption by soil is very important, as after soil adsorptive capacity is exceeded, the metal become potentially available for leaching. The objectives of this study were to analyze copper adsorption considering different properties of soil samples studied, to evaluate the influence of the $\mathrm{pH}$ level on copper adsorption and to verify if Langmuir and Freundlich isotherms describe adequately the copper adsorption. Solutions containing different copper concentrations $\left(6,10,20,40,60\right.$ e $\left.80 \mathrm{mg} \mathrm{L}^{-1}\right)$ were adjusted to $\mathrm{pH}$ 4.0, 5.0 and 6.0. The organic matter content and cation exchange capacity were the soil properties that most influenced copper adsorption. Langmuir and Freundlich models offered a good fit for the experimental data. Copper adsorption varied with soil $\mathrm{pH}$, with greater adsorption at $\mathrm{pH}$ 6.0.
\end{abstract}

Key words: micronutrients, isotherms, soil properties

1 UAEA/UFCG, Av. Aprígio Veloso 882, Bodocongó, CEP 58109-970, Campina Grande, PB. Fone: (83) 3310-1285. E-mail: Ihgarofalo@hotmail.com

2 PPGEAg/UFCG, Av. Aprígio Veloso 882. Fone: (83) 3310-1055. E-mail: mebbrito@yahoo.com.br; aureanp@yahoo.com.br; rossini_agro@yahoo.com.br; susaneribeiro@yahoo.com.br; robytabolka@yahoo.com.br 


\section{INTRODUÇÃO}

O elemento cobre (Cu), em virtude de atender aos critérios de essencialidade para as plantas, é classificado como micronutriente, porém quando presente em altas concentrações no solo, pode apresentar caráter tóxico. De acordo com a literatura, a concentração média do elemento no solo está em torno de $20 \mu \mathrm{g} \mathrm{g}^{-1}$ variando na faixa de 6 a $80 \mu \mathrm{g} \mathrm{g}^{-1}$ (Tavares \& Carvalho, 1992). Esta variação é resultado da aplicação, ao solo, de pesticidas, fertilizantes e, mais recentemente, de resíduos urbanos e industriais em decorrência do crescente interesse pela utilização desses resíduos na agricultura.

A dinâmica do cobre no solo é bastante complexa e altamente afetada por inúmeros fatores do meio, principalmente a composição química, física e mineralógica do solo, a quantidade de matéria orgânica e o pH (McBride et al., 1997). Conhecer o comportamento desse elemento nos solos, da mesma forma que o seu comportamento adsortivo pelos solos, torna-se fundamental para prever os impactos ambientais causados pelos mesmos, uma vez que os efeitos desfavoráveis de altas concentrações no meio ambiente estão relacionados com a capacidade dos solos em adsorver tais substâncias (Jordão et al., 2000).

O Cu pode ser adsorvido por atração eletrostática na dupla camada difusa (adsorção não específica) ou pela complexação de superfície (adsorção específica), processo no qual o elemento se liga à matriz do solo por ligações covalentes, reduzindo sua mobilidade (Selim, 1992); no primeiro caso, a capacidade de troca catiônica (CTC) do solo é fator decisivo, visto que representa a quantidade de sítios de troca nas interfaces coloidais do sistema, em que o Cu pode ser adsorvido; esta quantidade de sítios, por sua vez, é função da natureza dos colóides (minerais e orgânicos) presentes nos solos e do seu pH. Ambientes ácidos determinam maior mobilidade do $\mathrm{Cu}$ enquanto condições de $\mathrm{pH}$ acima de seis favorecem sua retenção (Hoog et al., 1993).

De acordo com Araújo \& Amaral Sobrinho (2000), as variáveis que mais contribuíram na adsorção do $\mathrm{Cu}$ nos solos estudados por eles, foram o carbono orgânico, a CTC efetiva, o pH, teor de argila e presença de óxido de alumínio. Silveira \& Alleoni (2003) e Nascimento \& Fontes (2004) também observaram que os teores de argila e de matéria orgânica foram as características mais bem relacionadas com a capacidade de adsorção do $\mathrm{Cu}$ por Latossolos.

Em relação à adsorção específica, da qual resulta a formação de moléculas estáveis com alta energia de ligação (Sparks, 1995), a matéria orgânica parece ser o fator mais importante, pois é ela que complexa todo o $\mathrm{Cu}$ da solução do solo. Em trabalho realizado por Araújo \& Amaral Sobrinho (2000), o Cu foi adsorvido predominantemente por adsorção específica na fração mineral dos solos estudados e por complexação com a matéria orgânica.

Uma das maneiras de se estudar o fenômeno de adsorção, inclusive do $\mathrm{Cu}$, é a partir do uso de isotermas de adsorção, as quais descrevem a relação entre a massa da substância adsorvida pela superfície sólida e a concentração da substância na solução de equilíbrio. Entre os modelos mais utilizados para se descrever a adsorção dos elementos, tem-se os de Langmuir e de Freundlich (Sposito, 1989). A teoria de Langmuir se baseia no fato da adsorção ocorrer em sítios uniformes com recobrimento em monocamada e afinidade iônica, independente da quantidade de material adsorvido (Atkins, 1994). O modelo de Freundlich considera a não uniformidade das superfícies reais e, quando aplicado ao solo, descreve bem a adsorção iônica dentro de certos limites de concentração mas acima deles apresenta dificuldade em considerar a quantidade de íons presentes no solo (Barrow, 1978). Apesar das limitações desses dois modelos, eles podem ser utilizados para predizer o comportamento dos metais nos solos.

Segundo Nascimento \& Fontes (2004), as isotermas de Langmuir e Freundlich se têm mostrado adequadas para o estudo de adsorção de vários elementos em solos brasileiros como, por exemplo, zinco, cobre, boro e cromo, dentre outros; no entanto, a isoterma de Freundlich foi a que melhor se ajustou aos dados de adsorção de $\mathrm{Cu}$ (Arias et al., 2005; 2006).

Objetivou-se, com este trabalho, analisar o comportamento da adsorção de $\mathrm{Cu}$ considerando-se as diferentes características de amostras de solo, avaliar a influência do pH na adsorção do Cu e verificar a adequação das equações de Langmuir e Freundlich para descrever a adsorção deste elemento.

\section{MATERIAL E MÉTODOS}

Selecionaram-se, para os ensaios, amostras de Plintossolo coletadas em quatro diferentes áreas da fazenda Abre o Olho, localizada a $4^{\circ} 30^{\prime} \mathrm{S}$ e $42^{\circ} 49^{\prime} \mathrm{W}$ entre os municípios de União e Miguel Alves, Estado do Piauí, com diferentes propriedades químicas e físicas (Tabela 1).

Amostras compostas da camada arável $(0-20 \mathrm{~cm}$ de profundidade), obtidas a partir de 15 amostras simples de solo, coletadas em cada área selecionada, foram destorroadas, secadas ao ar e passadas em peneiras de $2 \mathrm{~mm}$ de diâmetro de malha; em seguida, foram caracterizadas, física e quimicamente, de acordo com EMBRAPA (1997) conforme mostra os resultados da Tabela 1 .

Tabela 1. Propriedades químicas e físicas das amostras de Plintossolo coletas nas diferentes áreas

\begin{tabular}{|c|c|c|c|c|c|c|c|c|}
\hline \multirow{2}{*}{ Área } & Areia & Silte & Argila & \multirow{2}{*}{$\begin{array}{c}\mathrm{pH} \\
\mathrm{H}_{2} \mathrm{O}\end{array}$} & \multirow{2}{*}{$\begin{array}{l}\mathrm{MO}^{(1)} \\
\mathbf{g ~ k g}^{-1}\end{array}$} & $\mathbf{S}^{(2)}$ & CTC $^{(3)}$ & \multirow{2}{*}{$\begin{array}{c}\mathbf{V}^{(4)} \\
\%\end{array}$} \\
\hline & & $\mathrm{g} \mathrm{kg}^{-1}$ & & & & cmol & $\mathrm{dm}^{-3}$ & \\
\hline 1 & 379,0 & 345,0 & 276,0 & 7,33 & 0,16 & 9,99 & 10,28 & 97,11 \\
\hline 2 & 519,0 & 305,0 & 176,0 & 4,75 & 0,90 & 2.56 & 5,94 & 43,06 \\
\hline 3 & 623,0 & 231,0 & 146,0 & 6,57 & 0,32 & 3,76 & 4,56 & 82,62 \\
\hline 4 & 504,5 & 239,5 & 256.0 & 6.86 & 0.70 & 10,16 & 11.10 & 91.53 \\
\hline
\end{tabular}

(1) MO - matéria orgânica; (2) $\mathrm{S}$ - soma de bases trocáveis; ${ }^{(3)}$ CTC - capacidade de troca catiônica determinada a $\mathrm{pH} 7$; ${ }^{(4)} \mathrm{V}$ - porcentagem de bases trocáveis

As amostras de solo (2 g de TFSA), acondicionadas em tubos de centrífuga de $50 \mathrm{~mL}$, em duas repetições, foram agitadas durante $1 \mathrm{~h}$ a $140 \mathrm{rpm}$ e deixadas em repouso $24 \mathrm{~h}$, a temperatura ambiente $\left(22 \pm 2{ }^{\circ} \mathrm{C}\right)$ com as soluções de tratamento de $\mathrm{Cu}(20 \mathrm{~mL})$ de concentrações 6, 10, 20, 40, 60, $80 \mathrm{mg} \mathrm{L}^{-1}$ do elemento, na forma de $\mathrm{CuCl}_{2} 2 \mathrm{H}_{2} \mathrm{O}$ em 
0,01 M de NaCl. Para verificar o efeito do pH sobre a adsorção, o pH das suspensões solo-solução de tratamento foi ajustado a valores próximos de $\mathrm{pH}$ 4,0 \pm 0,1, pH 5,0 \pm 0,1 e pH 6,0 \pm 0,1, com soluções diluídas de $\mathrm{HCl}$ e $\mathrm{NaOH}$; após o período de repouso as suspensões foram filtradas e analisadas para $\mathrm{Cu}$ por espectrofotometria de absorção atômica. As concentrações de $\mathrm{Cu}$ adsorvidas foram consideradas a diferença entre aquelas inicialmente adicionadas e as remanescentes após o período de equilíbrio.

A partir dos dados obtidos experimentalmente se construíram isotermas de adsorção, plotando-se a quantidade de $\mathrm{Cu}$ adsorvido na ordenada e a concentração na solução de equilíbrio na abscissa. Obtiveram-se os coeficientes das equações de Freundlich e Langmuir a partir das Eqs. 1 e 2, a seguir:

a) Langmuir

$$
\frac{\mathrm{x}}{\mathrm{m}}=\frac{\mathrm{K}_{\mathrm{L}} \cdot \mathrm{C} \cdot \mathrm{b}_{\mathrm{L}}}{1+\mathrm{K}_{\mathrm{L}} \cdot \mathrm{C}}
$$

em que:

C - concentração de $\mathrm{Cu}$ na solução de equilíbrio, $\mathrm{mg} \mathrm{L}^{-1}$

$\mathrm{x} / \mathrm{m}$ - quantidade de $\mathrm{Cu}$ adsorvida, $\mathrm{mg} \mathrm{g}^{-1}$ de solo

$\mathrm{b}_{\mathrm{L}}$ - adsorção máxima, mg g-1

$\mathrm{K}_{\mathrm{L}}$ - constante relacionada à energia de ligação,

b) Freundlich $\mathrm{L} \mathrm{mg}^{-1}$ de $\mathrm{Cu}$ no solo e

$$
\frac{\mathrm{x}}{\mathrm{m}}=\mathrm{K}_{\mathrm{F}} \cdot \mathrm{C}^{\mathrm{b}}
$$

em que:

$\mathrm{x} / \mathrm{m}$ - quantidade de $\mathrm{Cu}$ adsorvida, $\mathrm{mg} \mathrm{g}^{-1}$ de solo

$\mathrm{C}$ - concentração de $\mathrm{Cu}$ na solução de equilíbrio, $\mathrm{mg} \mathrm{L}^{-1}$

$\mathrm{K}_{\mathrm{F}}$ - coeficiente de adsorção de Freundlich, L mg-1

$\mathrm{b}_{\mathrm{F}}(=1 / \mathrm{n})$ - estimativa do parâmetro de ajuste, adimensional

A significância estatística dos coeficientes de correlação para as isotermas foi o critério pelo qual seus dados foram testados.

\section{RESULTADOS E DISCUSSÃO}

\section{Características das amostras de solo}

As amostras de Plintossolo utilizadas neste trabalho apresentaram variações em suas propriedades físicas e químicas; os teores de argila variaram de 146 a $276 \mathrm{~g} \mathrm{~kg}^{-1}$ e os valores de $\mathrm{pH}$ de 4,75 a 7,33 correspondendo, segundo Lopes \& Guidollin (1989), a acidez média e alcalinidade fraca, respectivamente; os valores de matéria orgânica (MO) em todas as amostras foram classificados baixos e os valores de CTC variaram de 4,56 a 11,10 $\mathrm{cmol}_{\mathrm{c}} \mathrm{kg}^{-1}$, classificados médio e alto, respectivamente (Tabela 1 ).

\section{Efeito das características das amostras de solo na adsorção de cobre}

Para este estudo foram considerados apenas os dados de adsorção de $\mathrm{Cu}$ obtidos a pH $6 \pm 0,1$. Apesar das amostras de solo terem apresentado diferentes propriedades físicas e químicas (Tabela 1), os coeficientes de correlação mostram que os dados experimentais foram bem ajustados à isoterma de Langmuir, tanto pela equação original quanto pela linearizada (Tabela 2) corroborando com os resultados obtidos por Jordão et al. (2000), Sodré et al. (2001) e Nascimento \& Fontes (2004).

Tabela 2. Equações de Langmuir em suas formas original e linearizada e de Freundlich, correspondentes aos dados de adsorção de cobre nas amostras de Plintossolo coletas nas diferentes áreas, nos diferentes

\begin{tabular}{|c|c|c|c|c|}
\hline Área & $\mathrm{pH}$ & Isoterma & Equação & $r$ \\
\hline \multirow{7}{*}{1} & \multirow[t]{2}{*}{4} & $\mathrm{~L}$ & $\mathrm{x} / \mathrm{m}=(0,2709 \times 0,5710 \mathrm{C}) /(1+0,2709 \mathrm{C})$ & $0,9999 * *$ \\
\hline & & $\mathrm{L}$ & $\mathrm{C} / \mathrm{x} / \mathrm{m}=6,4625+1,7512 \mathrm{C}$ & $0,9779 * *$ \\
\hline & \multirow[t]{2}{*}{5} & $\mathrm{~L}$ & $\mathrm{x} / \mathrm{m}=(0,8090 \times 0,5889 \mathrm{C}) /(1+0,8090 \mathrm{C})$ & $0,9378^{* *}$ \\
\hline & & $\mathrm{L}$ & $\mathrm{C} / \mathrm{x} / \mathrm{m}=2,0989+1,698 \mathrm{C}$ & $0,9348^{* *}$ \\
\hline & \multirow[t]{3}{*}{6} & $\mathrm{~L}$ & $\mathrm{x} / \mathrm{m}=(2,3826 \times 0,7357 \mathrm{C}) /(1+2,3826 \mathrm{C})$ & 0,9631 ** \\
\hline & & $\mathrm{L}$ & $\mathrm{C} / \mathrm{x} / \mathrm{m}=0,5705+1,3593 \mathrm{C}$ & $0,9769 * *$ \\
\hline & & $\mathrm{F}$ & $\mathrm{x} / \mathrm{m}=0,407474 \mathrm{C}^{0,3814}$ & $0,9398^{* *}$ \\
\hline \multirow{7}{*}{2} & \multirow[t]{2}{*}{4} & $\mathrm{~L}$ & $\mathrm{x} / \mathrm{m}=(0,0891 \times 0,3433 \mathrm{C}) /(1+0,0891 \mathrm{C})$ & $0,9883^{\star \star}$ \\
\hline & & $\mathrm{L}$ & $\mathrm{C} / \mathrm{x} / \mathrm{m}=32,682+2,9129 \mathrm{C}$ & 0,9808 ** \\
\hline & \multirow[t]{2}{*}{5} & $\mathrm{~L}$ & $\mathrm{x} / \mathrm{m}=(0,2242 \times 0,4648 \mathrm{C}) /(1+0,2242 \mathrm{C})$ & 0,9730 ** \\
\hline & & $\mathrm{L}$ & $\mathrm{C} / \mathrm{x} / \mathrm{m}=9,609+2,1548 \mathrm{C}$ & $0,9271^{* *}$ \\
\hline & \multirow[t]{3}{*}{6} & $\mathrm{~L}$ & $\mathrm{x} / \mathrm{m}=(0,5524 \times 0,8173 \mathrm{C}) /(1+0,5524 \mathrm{C})$ & $0,9910^{* *}$ \\
\hline & & $\mathrm{L}$ & $\mathrm{C} / \mathrm{x} / \mathrm{m}=2,215+1,2235 \mathrm{C}$ & $0,8992^{*}$ \\
\hline & & $\mathrm{F}$ & $\mathrm{x} / \mathrm{m}=0,235831 \mathrm{C}^{0,5477}$ & $0,9731^{* *}$ \\
\hline \multirow{7}{*}{3} & \multirow[t]{2}{*}{4} & $\mathrm{~L}$ & $\mathrm{x} / \mathrm{m}=(0,3282 \times 0,4379 \mathrm{C}) /(1+0,3282 \mathrm{C})$ & $0,9999 * *$ \\
\hline & & $\mathrm{L}$ & $\mathrm{c} / \mathrm{x} / \mathrm{m}=6,9576+2,2838 \mathrm{C}$ & $0,9863^{* *}$ \\
\hline & \multirow[t]{2}{*}{5} & $\mathrm{~L}$ & $\mathrm{x} / \mathrm{m}=(0,4829 \times 0,5109 \mathrm{C}) /(1+0,4829 \mathrm{C})$ & $0,9999 * *$ \\
\hline & & $\mathrm{L}$ & $\mathrm{C} / \mathrm{x} / \mathrm{m}=4,0528+1,9573 \mathrm{C}$ & $0,9691^{* *}$ \\
\hline & \multirow[t]{3}{*}{6} & $\mathrm{~L}$ & $\mathrm{x} / \mathrm{m}=(1,4329 \times 0,6485 \mathrm{C}) /(1+1,4329 \mathrm{C})$ & $0,9999^{*}$ \\
\hline & & $\mathrm{L}$ & $\mathrm{C} / \mathrm{x} / \mathrm{m}=1,0762+1,5419 \mathrm{C}$ & $0,9842^{\star *}$ \\
\hline & & $\mathrm{F}$ & $\mathrm{x} / \mathrm{m}=0,229034 \mathrm{C}^{0,5081}$ & $0,9221^{* *}$ \\
\hline \multirow{7}{*}{4} & \multirow[t]{2}{*}{4} & $\mathrm{~L}$ & $\mathrm{x} / \mathrm{m}=(0,5654 \times 0,6074 \mathrm{C}) /(1+0,5654 \mathrm{C})$ & $0,9869^{* *}$ \\
\hline & & $\mathrm{L}$ & $\mathrm{C} / \mathrm{x} / \mathrm{m}=2,9114+1,6462 \mathrm{C}$ & 0,9930 ** \\
\hline & \multirow[t]{2}{*}{5} & $\mathrm{~L}$ & $\mathrm{x} / \mathrm{m}=(0,7569 \times 0,6621 \mathrm{C}) /(1+0,7569 \mathrm{C})$ & $0,9748^{* *}$ \\
\hline & & $\mathrm{L}$ & $\mathrm{C} / \mathrm{x} / \mathrm{m}=1,9954+1,5104 \mathrm{C}$ & $0,9692^{\star *}$ \\
\hline & \multirow[t]{3}{*}{6} & $\mathrm{~L}$ & $\mathrm{x} / \mathrm{m}=(2,2452 \times 0,83398 \mathrm{C}) /(1+2,2452 \mathrm{C})$ & 0,9861 ** \\
\hline & & $\mathrm{L}$ & $\mathrm{C} / \mathrm{x} / \mathrm{m}=0,5303+1,1907 \mathrm{C}$ & $0,9831^{* *}$ \\
\hline & & $\mathrm{F}$ & $\mathrm{x} / \mathrm{m}=0,48708 \mathrm{C}^{0,4434}$ & $0,9984^{* *}$ \\
\hline
\end{tabular}
valores de $\mathrm{pH}$

$\mathrm{L}, \mathrm{F}$, isotermas de Langmuir e Freundlich, respectivamente; * ${ }^{\star *}$ : significativo a 5 e $1 \%$, respectivamente

As relações entre os teores de Cu na solução de equilíbrio e os adsorvidos nas amostras de solo (Figura 1) mostram que o aumento na adsorção foi menos pronunciada a medida em que se aumentaram as concentrações do elemento adicionado a essas amostras, corroborando com Silveira \& Alleoni (2003). Segundo Petruzzelli et al. (1985) sempre que se aumenta a concentração do metal, mais sítios de adsorção vão sendo preenchidos dificultando, assim, a retenção do $\mathrm{Cu}$.

Observa-se, na Figura 1, que as amostras de solo são diferentes quanto à afinidade de adsorção do $\mathrm{Cu}$; no entanto, todas elas, para as baixas concentrações do elemento na solução de equilíbrio, apresentaram o tipo " $\mathrm{H}$ ” ( $\mathrm{H}=$ High) de isoterma, conforme classificação de Giles et al. (1974), 


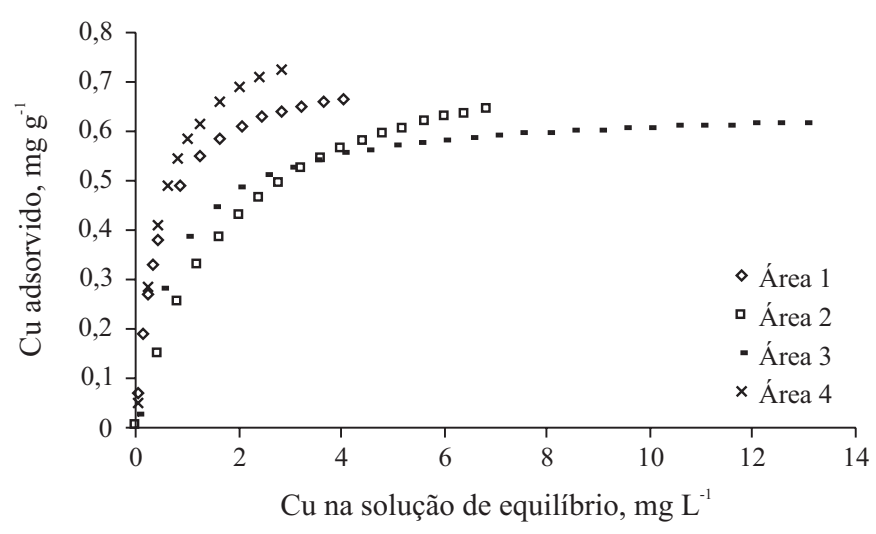

$\overline{\text { Figura 1. Relação entre as concentrações de Cu na solução de equilíbrio e }}$ as concentrações adsorvidas nas amostras de Plintossolo, a pH 6,0, estimadas pelos modelos de Langmuir

resultado da forte interação específica entre as partículas do solo e o $\mathrm{Cu}$. A afinidade das amostras de solo pelo Cu pode ser notada pelas concentrações remanescentes na solução de equilíbrio vindo, em ordem decrescente de afinidade, as amostras das áreas 4, 1, 3 e 2; esta mesma seqüência foi observada com os dados de adsorção de $\mathrm{Cu}$ a $\mathrm{pH} 4 \pm 0,1$ e pH $5 \pm 0,1$ (dados não apresentados). A afinidade de um solo pelo $\mathrm{Cu}$ é resultante de vários processos que interagem e são influenciados pelas propriedades dos solos como, por exemplo, $\mathrm{pH}$, teor de matéria orgânica, teor e tipo de argila e CTC, entre outras. No caso específico do presente trabalho, as amostras de solo com maior afinidade pelo $\mathrm{Cu}$, ou seja, aquelas correspondentes às áreas 4 e 1 apresentaram, em relação às demais, maior teor de argila, maior $\mathrm{pH}$ e maior CTC.

$\mathrm{O}$ parâmetro b da equação de Langmuir tem sido utilizado para estimar a capacidade máxima de adsorção (CMA) para vários elementos químicos presentes nos solos. A CMA de $\mathrm{Cu}$ mais elevada foi $0,8398 \mathrm{mg} \mathrm{g}^{-1}$, encontrada na amostra de solo da área 4, seguida de 0,8173, 0,7357 e $0,6485 \mathrm{mg} \mathrm{g}^{-1}$ encontradas nas amostras de solo das áreas 2, 1 e 3, respectivamente (Tabela 3). A textura do solo é a principal propriedade física que influencia na adsorção dos elementos químicos pelos solos; no entanto, no caso da amostra de solo da área 4, sua maior CMA deve estar relacionada ao seu teor de MO, uma vez que a mesma apresenta menor teor de argila que a amostra de solo da área 1. Segundo Araújo \& Amaral Sobrinho (2000), os atributos argila e carbono orgânico são as principais causas de variação da adsorção do $\mathrm{Cu}$.
Além do provável aumento do número de cargas negativas na superfície dos colóides da amostra de solo da área 2, provocado pela elevação do seu $\mathrm{pH}$, quando do equilíbrio a pH 6, ter favorecido a adsorção de $\mathrm{Cu}$, a quantidade de $\mathrm{MO}$ presente na referida amostra (Tabela 1) também deve ter sido o fator determinante para que a mesma apresentasse o segundo maior valor de CMA. De acordo com McLaren \& Crawford (1973) e Cavallaro \& McBride (1984) a MO tem elevada afinidade com o $\mathrm{Cu}$, especialmente sob condições ácidas, tendendo a formar complexos $\mathrm{Cu}-\mathrm{MO}$, o que pode provocar elevada capacidade de adsorção do elemento. Nascimento \& Fontes (2004) também observaram a influência da MO na adsorção de Cu em Latossolos. A amostra de solo da área 3 apresentou menor CMA decorrente, provavelmente, do fato da mesma apresentar menor teor de argila, menor teor de $\mathrm{MO}$ e, consequentemente, menor CTC, o que significa menor número de cargas negativas em seus colóides, em que o $\mathrm{Cu}$ poderia ser adsorvido.

\section{Efeito do pH na adsorção de cobre}

As amostras de solo estudadas, além de serem diferentes quanto à afinidade de adsorção do Cu (Figura 2), cada uma delas tem sua afinidade de adsorção variando com o pH do meio (Tabela 3); por exemplo, a amostra de solo da área 2 no $\mathrm{pH} 4$ e no pH 6, apresentou tipos "L" (L = Langmuir) e " $\mathrm{H}$ " de isotermas, correspondentes à menor e maior energia de ligação, respectivamente. A isoterma do tipo "L” se caracteriza por um decréscimo na inclinação da curva, sempre que os sítios disponíveis para a adsorção vão diminuindo; isto ocorre devido ao recobrimento da superfície de adsorção, o que indica, em baixas concentrações, que a superfície tem alta afinidade pela substância adsorvida, sendo que esta afinidade diminui em maiores concentrações (Dias et al., 2001).

Conforme a Figura 2, a adsorção do $\mathrm{Cu}$ pelas amostras de solo variou de acordo com o pH da suspensão solo-solução de tratamento, tendo-se observado maior adsorção com o pH 6,0 (Tabela 3). As concentrações de $\mathrm{Cu}$ adsorvidas por cada amostra de solo, quando no pH 4 e pH 5 foram semelhantes, corroborando com Jordão et al. (2000); no entanto, ao contrário do observado por esses autores, referidas amostras não apresentaram valores semelhantes de energia de ligação; deste modo, nos dois valores de $\mathrm{pH}$ examinados as amostras de solo adsorveram concentrações semelhantes de $\mathrm{Cu}$, porém não com a mesma energia.

$\mathrm{O}$ efeito do $\mathrm{pH}$ na adsorção de cátions metálicos resulta

Tabela 3. Parâmetros de ajuste das equações de Langmuir e Freundlich para dados de adsorção de cobre nas amostras de Plintossolo estudadas, com diferentes $\mathrm{pH}$

\begin{tabular}{|c|c|c|c|c|c|c|c|c|c|}
\hline \multirow{4}{*}{ Áreas } & \multicolumn{6}{|c|}{ Langmuir } & \multicolumn{3}{|c|}{ Freundlich } \\
\hline & \multicolumn{3}{|c|}{ Energia de Ligação $\left(\mathrm{K}_{\mathrm{L}}\right)$} & \multicolumn{3}{|c|}{ CMA $\left(b_{L}\right)$} & \multirow{2}{*}{$\begin{array}{c}\mathrm{K}_{\mathrm{F}} \\
\left(\mathrm{L} \mathrm{mg}^{-1}\right)\end{array}$} & \multirow[t]{2}{*}{$\mathbf{b}_{\mathrm{F}}$} & \multirow[t]{2}{*}{$\mathrm{n}$} \\
\hline & & $\mathrm{L} \mathrm{mg}^{-1}$ & & & $\mathrm{mg} \mathrm{g}^{-1}$ & & & & \\
\hline & pH 4 & pH 5 & pH 6 & pH 4 & pH 5 & pH 6 & & pH 6 & \\
\hline 1 & 0,2709 & 0,8090 & 2,3826 & 0,5710 & 0,5889 & 0,7357 & 0,4075 & 0,3814 & 2,6219 \\
\hline 2 & 0,0891 & 0,2242 & 0,5524 & 0,3433 & 0,4648 & 0,8173 & 0,2358 & 0,5477 & 1,8258 \\
\hline 3 & 0,3282 & 0,4829 & 1,4329 & 0,4379 & 0,5109 & 0,6485 & 0,2290 & 0,5081 & 1,9681 \\
\hline 4 & 0,5654 & 0,7569 & 2,2452 & 0,6074 & 0,6621 & 0,8398 & 0,4871 & 0,4434 & 2,2553 \\
\hline
\end{tabular}



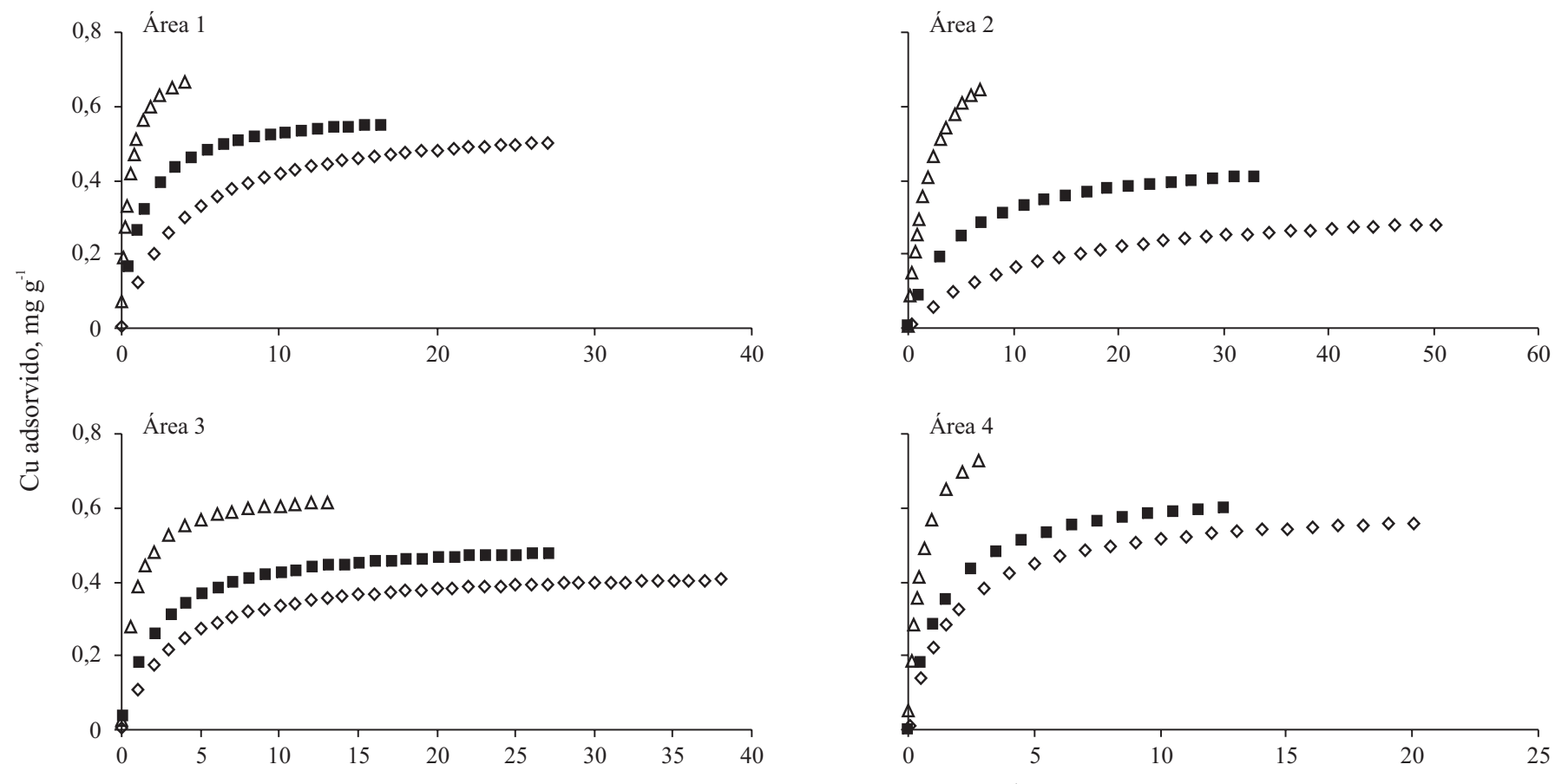

$\mathrm{Cu}$ na solução de equilíbrio, $\mathrm{mg} \mathrm{L}^{-1}$

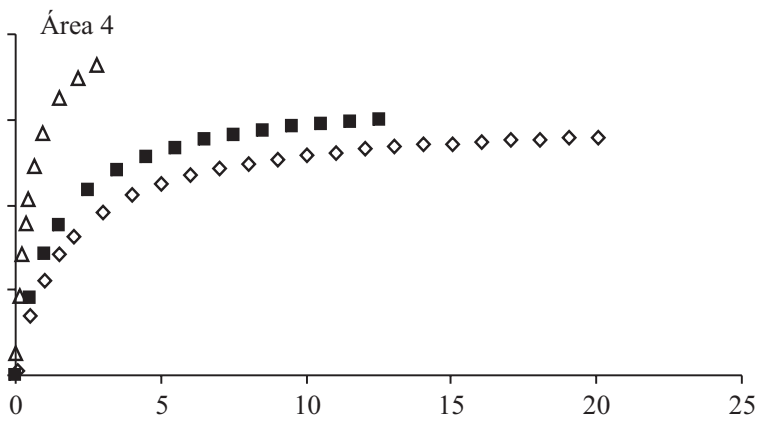

$\diamond \mathrm{pH} 4 \quad$ - $\mathrm{pH} 5 \quad \Delta \mathrm{pH} 6$

Figura 2. Relação entre os teores de Cu na solução de equilíbrio e os adsorvidos nas amostras de Plintossolo das quatro áreas, em função do pH

principalmente das mudanças da carga protônica líquida das partículas do solo. Com o aumento do $\mathrm{pH}$, esta carga decresce para valores mais negativos, resultando na atração eletrostática do solo pelo íon metálico (Alcântara \& Camargo, 2001); isto poderia explicar o aumento da adsorção de $\mathrm{Cu}$ observado com a elevação do pH na amostra de solo da área 2, cujo pH original estava em torno de 4,75 (Figura 2). Para as amostras de solo das demais áreas, cujos valores de $\mathrm{pH}$ estavam em torno de 7 , na medida em que se diminuiu o $\mathrm{pH}$ da suspensão solo/solução de tratamento, ocorreu diminuição da CMA do Cu (Tabela 3), o que já era previsto, uma vez que deve ter diminuído o número de cargas elétricas negativas na superfície dos colóides dos solos; todavia, analisando-se os dados sob o aspecto da disponibilidade de $\mathrm{Cu}$ para as plantas ou sob o aspecto de contaminação ambiental, a amostra de solo da área 2, em seu pH original, por apresentar menor energia de ligação e menor adsorção de $\mathrm{Cu}$, seria aquela na qual o elemento, presente no solo, ficaria mais disponível, tanto para ser absorvido pelas plantas como para ser lixiviado, podendo contaminar o lençol freático.

\section{Ajuste das isotermas de adsorção}

Para este estudo se consideraram também apenas os dados de adsorção de $\mathrm{Cu}$ pelas amostras a pH 6,0 (Tabelas 2 e 3).

Os altos coeficientes de correlação das equações correspondentes aos modelos de Langmuir e Freundlich obtidos no presente trabalho (Tabela 2) indicam que tais modelos se adequaram aos valores de $\mathrm{Cu}$ adsorvidos pelas amostras de solo estudadas concordando com Araújo \& Amaral Sobrinho (2000), Sodré et al. (2001), Silveira \& Alleoni (2003) e Nascimento \& Fontes (2004); todavia, Araújo \& Amaral Sobrinho (2000) e Arias et al. (2006) ressaltam que a adsorção de Cu é mais bem representada pela equação de Freundlich justificando o fato pela heterogeneidade da superfície de adsorção.

Os valores obtidos em laboratório, de $\mathrm{Cu}$ adsorvido aos solos e as curvas obtidas a partir dos valores estimados pelos modelos de Langmuir e Freundlich, são apresentados na Figura 3.

Para as doses até $10 \mathrm{mg} \mathrm{L}^{-1}$ de $\mathrm{Cu}$ adicionadas aos solos, os dois modelos estimaram valores semelhantes aos obtidos experimentalmente; para as demais doses utilizadas, o comportamento dos modelos foi diferente em relação às áreas de estudo. Observa-se que, com exceção da área 3, nas demais áreas e para as doses até $60 \mathrm{mg} \mathrm{L}^{-1}$, os valores de $\mathrm{Cu}$ adsorvido estimados pelos modelos ou foram semelhantes aos valores experimentais ou foram superestimados; para a dose mais alta, $80 \mathrm{mg} \mathrm{L}^{-1}$, nas áreas 1 e 2, os valores de $\mathrm{Cu}$ adsorvido foram subestimados pelos modelos de Langmuir e Freundlich; nas áreas 3 e 4 continuou sendo subestimado pelo modelo de Langmuir; no entanto, foi superestimado pelo de Freundlich na área 3 e semelhante ao observado na área 4. Em geral, as concentrações de $\mathrm{Cu}$ adsorvido estimadas pelo modelo de Langmuir, até a dose $60 \mathrm{mg} \mathrm{L}^{-1}$, foram maiores que aquelas estimadas pelo modelo de Freundlich; a partir daí, houve uma inversão de comportamento, ou seja, para a dose mais alta utilizada o modelo de Freundlich estimou valores maiores que aqueles obtidos por Langmuir. Com relação ao ponto de inversão, nota-se comportamento diferenciado entre as áreas estudadas, sendo que este ocorreu na área 1 entre 3 e $4 \mathrm{mg} \mathrm{L}^{-1}$; nas áreas 2 e 3 entre 6 e $7 \mathrm{mg} \mathrm{L}^{-1}$, enquanto na área 4 a inversão se deu entre 2 e $3 \mathrm{mg} \mathrm{L}^{-1}$ de $\mathrm{Cu}$ da solução de equilíbrio. $\mathrm{O}$ fato dos valores das concentrações de $\mathrm{Cu}$ na solução de equilíbrio serem diferentes, a partir dos quais o modelo de 

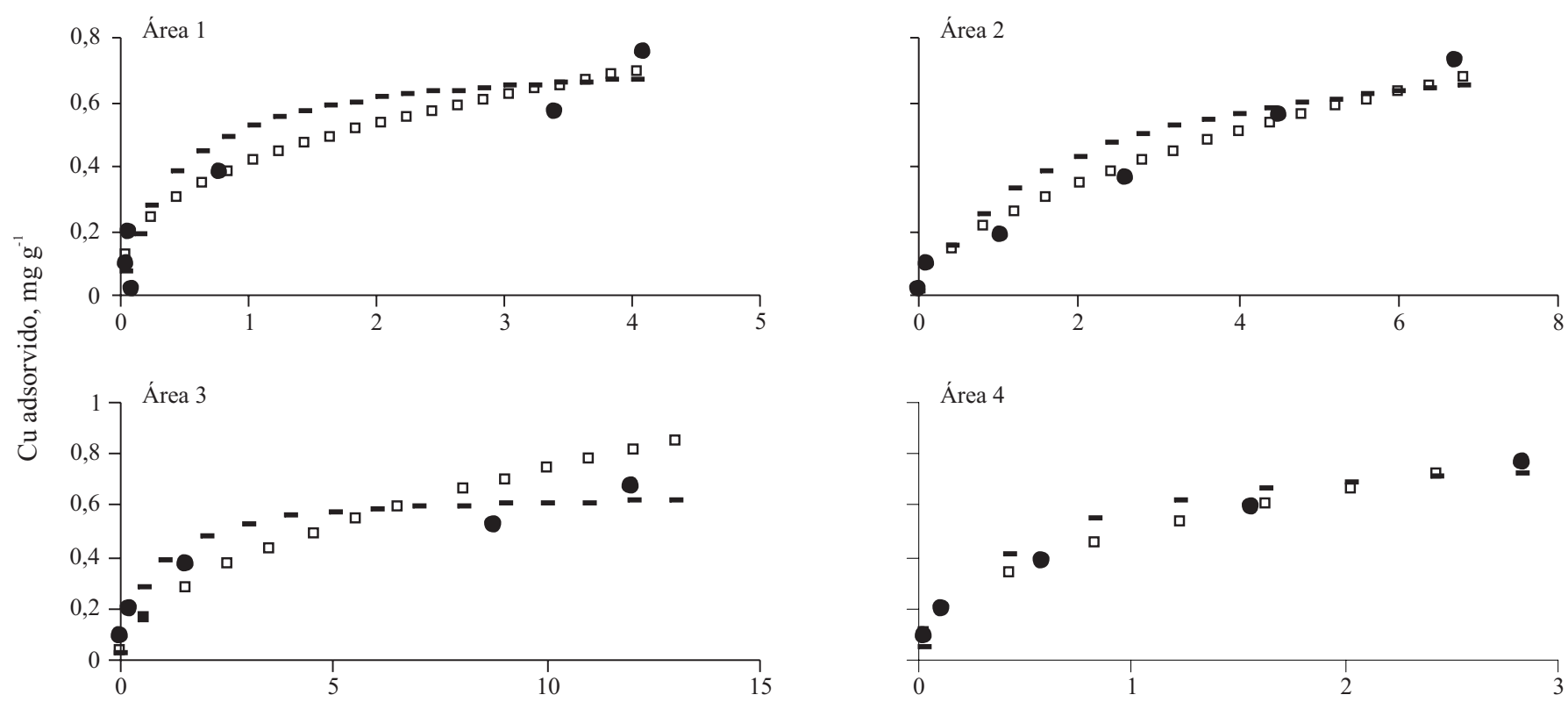

$\mathrm{Cu}$ na solução de equilíbrio, $\mathrm{mg} \mathrm{L}^{-1}$

- Langmuir

口 Freundlich

- Série3

Figura 3. Relação entre as concentrações de Cu na solução de equilíbrio e as concentrações adsorvidas nas amostras de Plintossolo, estimados pelos modelos de Langmuir e Freundlich, e valores obtidos experimentalmente

Freundlich estima maiores valores de $\mathrm{Cu}$ adsorvido, está relacionado com a capacidade máxima de adsorção do elemento pelos solos. Relata-se que, quanto maior for a capacidade de adsorver o elemento pelo solo, menor será sua concentração na solução de equilíbrio, sendo que neste trabalho a maior adsorção foi observada na área 4 e as menores nas áreas 1 e 3.

Os valores para o coeficiente $K_{L}$, relativos à energia de ligação, apresentaram-se mais elevados para os solos das áreas 1 e 4, indicando uma retenção mais forte de cobre pelas suas amostras. Os valores de $b_{F}$ variaram de 0,38 na área 1 , a 0,54 na área 2, estando de acordo com os valores encontrados por Arias et al. (2005), trabalhando em solos cultivados. Segundo Cunha et al. (1994), os valores do $b_{F}$ seguem, aproximadamente, a mesma tendência de $\mathrm{K}_{\mathrm{L}}$; todavia no presente trabalho isto foi observado entre os valores de $K_{L}$ e $n$.

Sposito (1989), estudando as derivações da equação de Freundlich para íons trocáveis em solos, observou uma relação qualitativa entre o parâmetro $n\left(b_{F}=1 / n\right)$ e a distribuição dos sítios energéticos na fração dispersa dos colóides do solo. Quando $n \neq 1$, a distribuição desses sítios tende a variar com a densidade de adsorção. Os valores de n para as amostras de solo estudadas apresentaram valores acima de 1 (Tabela 3), indicando a presença de sítios altamente energéticos e sugerindo que estes são os primeiros a serem ocupados pelo Cu (Inskeep \& Baham, 1983).

A partir dos parâmetros de ajuste das equações de Langmuir e Freundlich e de algumas características das amostras de solo estudadas, fez-se uma análise de correlação simples (Tabela 4). Apesar do pequeno número de dados, encontrouse correlação entre $K_{F}$ e CTC, cujo coeficiente 0,98 foi significativo a $5 \%$ de probabilidade, corroborando com Silveira \& Alleoni (2003), Nascimento \& Fontes (2004) e Arias et al. (2005); isto implica dizer que a adsorção de Cu nessas amostras foi influenciada pela CTC das mesmas uma vez que, segundo Sodré et al. (2001), o $K_{F}$ pode sugerir a adsorção do íon no solo.

Tabela 4. Matriz de correlação simples entre os parâmetros das isotermas de Langmuir e Freundlich com algumas características das amostras de Plintossolo estudadas

\begin{tabular}{lrrrrrr}
\hline Características das & \multicolumn{2}{c}{ Langmuir } & & \multicolumn{2}{c}{ Freundlich } \\
\cline { 2 - 3 } \cline { 6 - 7 } amostras de solo & \multicolumn{1}{c}{$\mathbf{K}_{\mathrm{L}}$} & $\mathbf{b}_{\mathrm{L}}$ & & $\mathbf{K}_{\mathbf{F}}$ & $\mathbf{b}_{\mathbf{F}}$ \\
Teor de argila & $0,80 \mathrm{~ns}$ & $0,45 \mathrm{~ns}$ & & $0,91 \mathrm{~ns}$ & $-0,89 \mathrm{~ns}$ \\
pH & $0,95 \mathrm{~ns}$ & $-0,34 \mathrm{~ns}$ & & $0,64 \mathrm{~ns}$ & $-0,87 \mathrm{~ns}$ \\
Teor de M0 & $-0,61 \mathrm{~ns}$ & $0,75 \mathrm{~ns}$ & & $-0,11 \mathrm{~ns}$ & $0,64 \mathrm{~ns}$ \\
CTC & $0,80 \mathrm{~ns}$ & $0,55 \mathrm{~ns}$ & & $0,98 *$ & $-0,83 \mathrm{~ns}$ \\
\hline
\end{tabular}

* significativo a $5 \%$ de probabilidade ; ns não significativo

\section{CONCLUSÕES}

1. Os teores de matéria orgânica e a capacidade de troca catiônica das amostras de Plintossolo foram as propriedades que mais influenciaram na adsorção de cobre.

2. Os modelos de Langmuir e Freundlich se mostraram igualmente capazes para descrever o comportamento do cobre nas diferentes amostras de solo estudadas.

3. A adsorção de cobre variou em função do $\mathrm{pH}$ tendo ocorrido maior adsorção no pH 6,0.

\section{LITERATURA CITADA}

Alcântara, M. A. K.; Camargo, O. A. Isotermas de adsorção de Freundlich para crômio (III) em latossolos. Scientia Agrícola, v.58, n.3, p.567-572, 2001. 
Araújo, W. S.; Amaral Sobrinho, N. M. B. Influência das propriedades físicas e químicas de solos intemperizados na adsorção de chumbo, cobre e zinco. Floresta e Ambiente, v.7, n.1, p.167-180, 2000.

Arias, M.; Pérez-Novo, C.; López, E.; Soto, B. Competitive adsorption and desorption of copper and zinc in acid soils. Geoderma, v.133, n.3-4, p.151-159, 2006.

Arias, M.; Pérez-Novo, C.; Osorio, F.; López, E.; Soto, B. Adsorption and desorption of copper and zinc in the surface layer of acid soils. Colloid and Interface Science, v.288, n.1, p.21-29, 2005.

Atkins, P. W. Physical chemistry. 5.ed. Oxford: Oxford University Press, 1994. 279p.

Barrow, N. J. The description of phosphorus adsorption curves. Journal of Soil Science, v.29, p.447-462, 1978.

Cavallaro, N.; Mcbride, M. B. Zinc and copper sorption and fixation by an acid soil clay: Effect of selective dissolution. Soil Science Society of America Journal, v.48, n.4, p.1050-1054, 1984.

Cunha, R. C. A.; Camargo, O. A.; Kinjo, T. Aplicação de três isotermas na adsorção de zinco em Oxissolos, Alfissolos e Ultissolos. Revista Brasileira de Ciência do Solo, v.18, n.1, p.5-20, 1994.

Dias, N. M. P.; Alleoni, L. R. F.; Casagrande, J. C.; Camargo, O. A. Isotermas de adsorção de cádmio em solos ácricos. Revista Brasileira de Engenharia Agrícola e Ambiental, v.5, n.2, p.229-234, 2001.

EMBRAPA - Empresa Brasileira de Pesquisa Agropecuária. Centro Nacional de Pesquisa de Solos. Manual de métodos de análise de solo. Rio de Janeiro: EMBRAPA, 1997. 212p.

Giles, C. H.; Smith, D.; Huitson, A. A general treatment and classification of the solute adsorption isotherm. I. Theoretical. Journal of Colloid and Interface Science, v.47, n.2, p.755-765, 1974.

Hoog, D. S.; McLaren, R. G.; Swift, R. S. Soil Science Society American Journal, v.57, n.2, p.361-366, 1993.

Inskeep, W. P.; Baham, J. Adsorption of Cd(II) and Cu(II) by NaMontmorillonite at low surface coverage. Soil Science Society American Journal, v.47, n.3, p.660-665, 1983.
Jordão, C. P.; Alves, N. M.; Pereira, J. L.; Bellato, C. R.; Alvarez, V. H. Adsorção de íons $\mathrm{Cu}^{2+}$ em Latossolo Vermelho-Amarelo Húmico. Química Nova, v.23, n.1, p.5-11, 2000.

Lopes, A. S.; Guidolin, J. A. Interpretação de análise do solo: conceitos e aplicações. 2.ed. São Paulo: Comitê de Pesquisa/ Técnico/ANDA, 1989. 64p.

McBride, M.; Sauvé, S.; Hendershot, W. Solubility control of $\mathrm{Cu}$, $\mathrm{Zn}, \mathrm{Cd}$ e $\mathrm{Pb}$ in contamined soils. European Journal of Soil Science, v.48, n.2, p.337-346, 1997.

McLaren, R. G.; Crawford, D. V. 1973. Studies of soil copper. II. The specific adsorption of copper by soils. European Journal of Soil Science, v.24, n.4, p.443-452, 1973.

Nascimento, C. W. A.; Fontes, R. L. F. Correlação entre características de latossolos e parâmetros de equações de adsorção de cobre e zinco. Revista Brasileira de Ciência do Solo, v.28, n.6, p.965-971, 2004.

Petruzzelli, G.; Guidi, G.; Lubrano, L. Ionic strength effect on heavy metal adsorption by soil. Communications in Soil Science and Plant Analysis, v.16, p.971-986, 1985.

Selim, H. M. Modelling the transport and retention of inorganics in soils. Advances in Agronomy, v.47, p.331-384, 1992.

Silveira, M. L. A.; Alleoni, L. R. F. Copper adsorption in tropical oxisols. Brazilian Archives of Biology and Technology. v.46, n.4, p.529-536, 2003.

Sodré, F. F.; Lenzi, E.; Costa, A. C. S. Utilização de modelos físicos-químicos de adsorção no estudo do comportamento do cobre em solos argilosos. Química Nova, v.24, n.3, p.324-330, 2001.

Sparks, D. L. Sorption phenomena on soils. In: Sparks, D. L. (ed.). Environmental soil chemistry. San Diego: John Wiley, p.99-139, 1995.

Sposito, G. The chemistry of soils. New York: Oxford University Press, 1989. 277p.

Tavares, T. M.; Carvalho, F. M. Avaliação de exposição de populações humanas a metais pesados no ambiente: exemplos do Recôncavo Baiano. Química Nova, v.15, n.2, p.147-154, 1992. 